\title{
Association of anthropometric measures with kidney disease progression and mortality: a retrospective cohort study of pre-dialysis chronic kidney disease patients referred to a specialist renal service
}

Emma Davis $^{1 *}$ D , Katrina Campbell ${ }^{1,2}$, Glenda Gobe ${ }^{1}$, Carmel Hawley ${ }^{1,2,3}$, Nicole Isbel ${ }^{1,2}$ and David W. Johnson ${ }^{1,2,3}$

\begin{abstract}
Background: Although elevated body mass index (BMI) is a predictor of better clinical outcomes in dialysis patients, the evidence in pre-dialysis chronic kidney disease (CKD) is conflicting. Clinical measures of central obesity may be better prognostic indicators, although investigation has been limited. The aim of this study was to assess the predictive value of anthropometric measures for kidney failure progression and mortality in stage 3-4 CKD.

Methods: The study included newly referred stage 3-4 CKD patients at a single centre between 1/1/2008 and 31/12/2010. The associations between clinical measures of obesity (BMI, waist circumference [WC] and conicity index [Conl]) and time to a composite primary outcome of doubling of serum creatinine, commencement of renal replacement therapy or mortality were evaluated using the Kaplan-Meier method and multivariable Cox regression models.

Results: Over a median follow-up period of 3.3 years, 229 (25.4\%) patients of a total population of 903 experienced the composite primary renal outcome. When compared to normal BMI $\left(18.5-24.9 \mathrm{~kg} / \mathrm{m}^{2}, n=174\right)$, the risk of the composite primary outcome was significantly lower in both the overweight (BMI $25-29.9 \mathrm{~kg} / \mathrm{m}^{2}, n=293$; adjusted hazard ratio [HR]

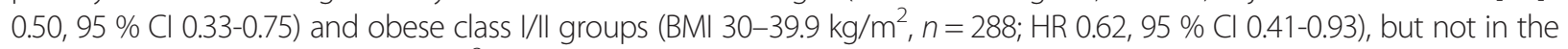
obese class III group (BMI $\left.\geq 40 \mathrm{~kg} / \mathrm{m}^{2}, n=72 ; \mathrm{HR} 0.94,95 \% \mathrm{Cl} 0.52-1.69\right)$. All-cause mortality was also lower in the overweight group (HR 0.50, $95 \% \mathrm{Cl} 0.30-0.83$ ). WC and Conl were not associated with either the composite primary outcome or mortality.
\end{abstract}

Conclusion: BMl in the overweight range is associated with reduced risks of kidney disease progression and all-cause mortality in stage 3-4 CKD. WC and Conl were not independent predictors of these outcomes in this population.

Keywords: Anthropometry, Body mass index, Chronic kidney disease, Conicity index, Mortality, Waist circumference

\footnotetext{
*Correspondence: emma.davis@uqconnect.edu.au

${ }^{1}$ Centre for Kidney Disease Research, School of Medicine, University of

Queensland, Translational Research Institute, Brisbane, QLD, Australia

Full list of author information is available at the end of the article
} 


\section{Background}

Chronic kidney disease (CKD) is a growing public health problem, with more than $10 \%$ of the adult population in both the United States [1] and Australia [2] estimated to have stage 1-4 CKD. Targeting modifiable lifestyle factors, such as obesity, has been frequently recommended as a first line strategy for reducing the risks of kidney disease progression and cardiovascular disease (CVD) in patients with CKD [3-5]. However, whilst obesity has been identified as a risk factor for new onset kidney disease [6] and mortality in the general population [7], the evidence in CKD is conflicting. Moreover, a recent cohort study of 453,946 United States veterans with an estimated glomerular filtration rate $($ eGFR $)<60 \mathrm{ml} / \mathrm{min}$ per $1.73 \mathrm{~m}^{2}$ observed a consistent, U-shaped association between BMI and the outcomes of kidney disease progression and mortality, with the best outcomes observed in overweight and mildly obese subjects [8]. This risk factor paradox has been deemed by some as "reverse causation", implying that there is unintentional illness-related weight loss which contributes to higher mortality [9].

Despite being a clinical tool which is widely used to assess obesity, BMI is an unreliable measure of body fat content in patients with CKD [10]. This measure is unable to differentiate between muscle and fat amount and distribution, and, while having reasonable correlation with body fat percentage, BMI has poor sensitivity for diagnosing obesity [11]. These limitations are particularly concerning in light of the increasing evidence that abdominal obesity is a key contributor to the health risks associated with obesity [12].

Visceral adiposity is associated with metabolic abnormalities and a pro-inflammatory state, which is linked with insulin resistance and an atherogenic lipoprotein profile [13]. Clinical measures that more precisely evaluate central obesity, such as waist circumference (WC) and conicity index (ConI) [14], have been more strongly associated with clinical outcomes than BMI in the general population [15] and in dialysis populations [16-18]. Although its prognostic value in non-dialysis CKD patients has not been well established,[19, 20] ConI, which adjusts waist circumference for height and weight, has been linked to a number of risk factors for metabolic syndrome [14] and kidney disease progression, including proteinuria and systemic inflammation [21]. It has also been linked to inflammation [22], and poor nutritional status resulting in an increased risk of mortality in haemodialysis patients [18].

The aim of this study was to investigate the associations between baseline anthropometric measures of body size (BMI, WC and ConI) and the clinical outcomes of kidney disease progression and all-cause mortality in incident Australian adults with stage 3-4 CKD referred to a specialist renal service. It was hypothesised that obesity markers that were more specific for central obesity, particularly waist circumference, would better predict progression to the composite renal endpoint and death.

\section{Methods}

The protocol for this retrospective observational cohort study was approved by Metro South Human Research Ethics Committee (HREC/10/QPAH/71) and University of Queensland School of Medicine Low Risk Ethics Committee (2014-SOMILRE-0094). General patient consent was attained at initial referral for use of clinical data in the Princess Alexandra Hospital (PAH) Nephrology Database.

\section{Study population}

The study population consisted of patients with CKD who were referred by general practitioners or specialists to the PAH Nephrology Outpatient Department between 1 January 2008 and 31 December 2010 (catchment population approximately 1 million or $23 \%$ of Queensland's population). Patients included those over the age of 18 years with an eGFR of $15-59 \mathrm{~mL} / \mathrm{min} / 1.73 \mathrm{~m}^{2}$ whose clinical data were recorded in the PAH Nephrology Database. Patients were excluded if they had no recorded clinical or laboratory data from within three months of initial referral visit.

\section{Data collection}

Baseline patient data from the initial visit were obtained from the PAH Nephrology Database and electronic medical records. Recorded variables included demographics, cause of kidney disease, comorbidities, medications, anthropometric measures (weight, waist circumference, height), blood pressure and laboratory values (serum creatinine, proteinuria, haemoglobin level, serum cholesterol). Height, weight and WC were measured by trained health practitioners using a standardised protocol. WC was measured at the midpoint between the lower margin of the last palpable rib and the top of the iliac crest. BMI was calculated as weight $(\mathrm{kg})$ divided by height $(\mathrm{m})$ squared and was categorised based on the World Health Organisation classification: underweight $\left(<18.5 \mathrm{~kg} / \mathrm{m}^{2}\right)$, normal (18.5-24.9 kg/m²), overweight $\left(25-29.9 \mathrm{~kg} / \mathrm{m}^{2}\right)$, obese class I/II $\left(30-39.9 \mathrm{~kg} / \mathrm{m}^{2}\right)$ and obese class III $\left(\geq 40 \mathrm{~kg} / \mathrm{m}^{2}\right)$. WC was divided into tertiles, stratified by gender. ConI was calculated as $\frac{W C(m)}{0.109 \times \sqrt{\frac{\text { weight }(\mathrm{kg})}{\text { height }(m)}}}$ and divided into tertiles.

Serum creatinine (traceable to isotopic dilution mass spectrometry) was measured by the Jaffe rate method using a Beckman DxC800 general chemistry analyser (Beckman Coulter, Brae, CA, USA). The baseline serum creatinine value from the initial referral visit was used to calculate an eGFR using the CKD-Epidemiology Collaboration (CKD-EPI) calculation [23]. Proteinuria was assessed using urine albumin-to-creatinine ratio (ACR), 
urine protein-to-creatinine ratio (PCR) or total protein in a 24-h urine collection and was categorised as normo, micro- or macroproteinuria according to the recommendations of the Kidney Check Australia Taskforce [24]. Urine protein was measured by immunoassay turbidimetric method and urine protein was measured by pyrogallol red and molybdate method using a Beckman DxC800 general chemistry analyser.

\section{Study outcomes}

Patients were followed until death, loss to follow up, or 31 July 2013, whichever came first. The primary outcome of interest in the study was a composite outcome of: 1) doubling of serum creatinine; 2) initiation of renal replacement therapy (RRT); and 3) all-cause mortality. Creatinine values were followed through until 31 July, 2013 to determine if there had been an increase of more than two times the serum creatinine from initial referral, confirmed on two readings at least four weeks apart. RRT was considered as any form of dialysis or kidney transplantation. Patients who were lost to follow-up in the PAH database were cross referenced with the Australia and New Zealand Dialysis and Transplant (ANZDATA) Registry for outcome data. The ANZDATA Registry has complete capture of all patients in Australia and New Zealand who have commenced RRT since 1963 (www.anzdata.org.au).

\section{Statistical analysis}

Results were expressed as frequencies and percentages for categorical variables, mean \pm standard deviation for continuous normally distributed variables, and median [interquartile range; IQR] for continuous variables that were not normally distributed. Categorical data were compared using chi-square tests. Continuous normally distributed data were compared using two tailed unpaired t-tests. Continuous non-normally distributed data were compared using Mann-Whitney tests. The association between anthropometric indices and time to either the composite primary end-point or mortality were evaluated by Kaplan Meier and multivariable Cox proportional hazards model analyses. Each anthropometric index was analysed as a categorical variable in the primary analysis and as a continuous variable in a sensitivity analysis. For the multivariable analyses, several models were examined: Model 1, adjusted for age (continuous); Model 2, adjusted for age, gender and race (Caucasian vs. non-Caucasian); and Model 3 included age, gender, race, cause of CKD (categorised based on the Study of Heart and Renal Protection [SHARP] trial [25]: diabetic nephropathy, glomerulonephritis, cystic kidney disease and other), eGFR, proteinuria, and presence of diabetes as a comorbidity. The data was further analysed with stratification by gender. Patients with missing data were excluded from multivariable analysis. $P$-values of $<0.05$ were considered significant. Statistical analyses were performed using SPSS Statistics version 21.

\section{Results \\ Baseline characteristics}

Of 1070 adult patients with CKD referred to the centre between 2008 and 2010, a total of 903 patients were included in the study (Fig. 1). Their baseline characteristics are shown in Table 1.

\section{Primary composite end-point}

During a median follow-up of 3.3 years, 229 patients (25.4\%) reached a composite primary outcome, including $15.7 \%$ who died, $8.3 \%$ who experienced a doubling of their serum creatinine and $1.3 \%$ who commenced RRT as the initial event. The median time to the primary end-point was 1.81 years.

On univariable analysis, patients with a BMI in the overweight range (hazard ratio [HR] 0.565, $95 \%$ CI 0.39 $0.81, p<0.01$ ) and in the class I/II obese range (HR 0.63, $95 \%$ CI 0.44-0.90) experienced a lower hazard of the primary end-point compared with normal weight individuals (Fig. 2, Table 2). Similar results were observed following multivariable Cox proportional hazards model analysis (Table 2, Fig. 3).

WC did not show a significant association with the primary outcome as either a continuous or a categorical variable in univariable analysis (Fig. 2, Table 2). Multivariable models also failed to identify a significant relationship between WC and the primary end-point.

In univariable survival analysis, increasing ConI was predictive of a higher risk of the composite outcome (Fig. 2, Table 2). Using ConI as a continuous variable, each 0.1 unit increase in ConI was associated with a $28 \%$ increased risk of progression to the composite outcome (HR, 1.278; $95 \%$ CI, 1.07-1.53; $P<0.01$.

\section{Mortality}

The relationship between different anthropometric measures and all-cause mortality are shown in Table 3. Those patients in the overweight BMI category had a lower risk of mortality. There was evidence of a U-shaped association, with $\mathrm{BMI} \geq 40 \mathrm{~kg} / \mathrm{m}^{2}$ showing increasing risk of mortality (Fig. 3).

There was no direct relationship between WC and all-cause mortality in either univariable or multivariable analysis.

ConI was also predictive of mortality (HR 1.413, $95 \%$ CI 1.13-1.76, $P<0.01$ for each 0.1 unit increase in ConI). This association remained significant when adjusted for demographics but not when fully-adjusted. Increasing ConI tertiles were also predictive of a greater risk of death in the crude model, although significance was lost following adjustment (Table 3). 


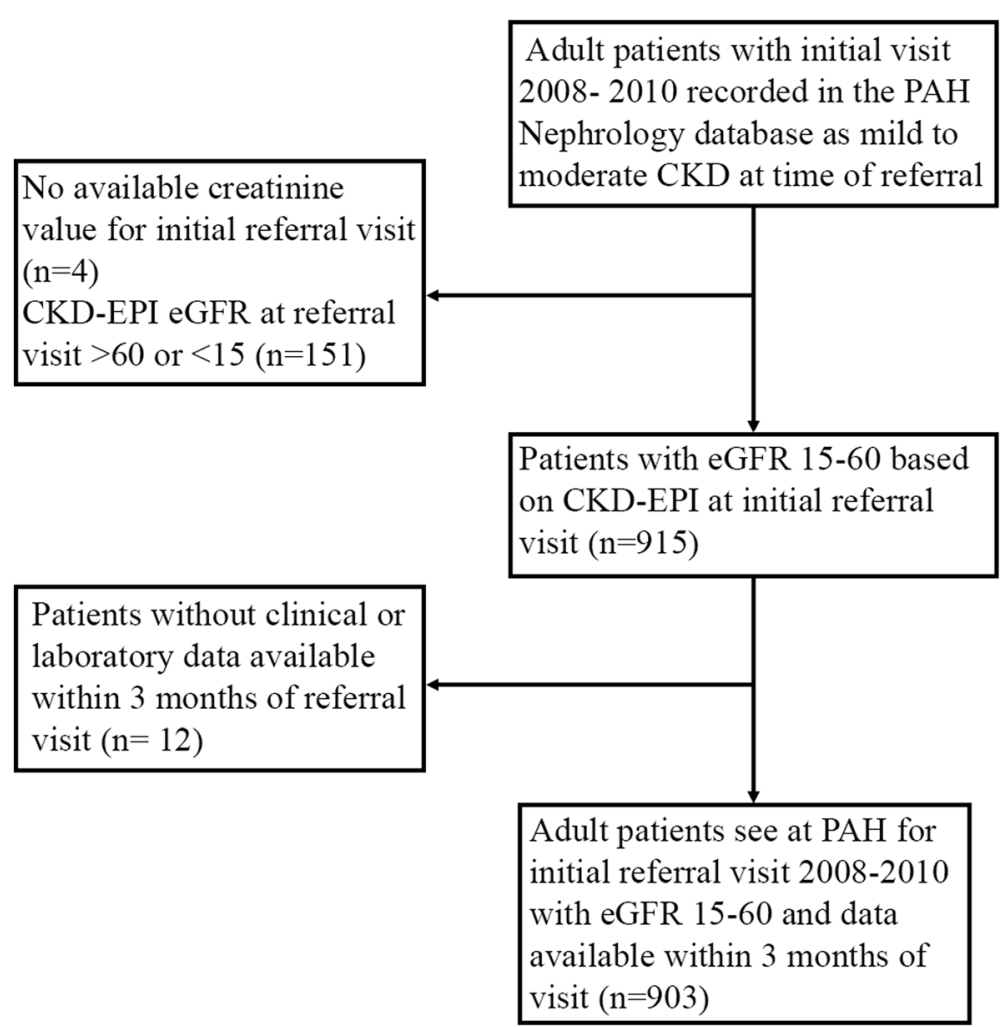

Fig. 1 Derivation of the Study Cohort. PAH, Princess Alexandra Hospital; CKD, chronic kidney disease; CKD-EPI, CKD-Epidemiology Collaboration [30]; eGFR, estimated glomerular filtration rate

\section{Gender Sub-analysis}

When stratifying the data by gender (see Additional file 1: Table S1 and Table S2), a U-shaped association between BMI and the composite outcome was maintained for crude and multivariable analysis in both males and females. Model 2 and 3 lost statistical significance in the female group. The U-shaped association between BMI category and mortality was notably stronger in males over females, where the relationship was not significant at any level. For the composite outcome WC and ConI was not associated with the outcome for males or females in any model.

\section{Discussion}

In the current study, BMI values in the overweight and class I/II obese range were shown to be associated with lower hazards of both the composite primary renal outcome and all-cause mortality in a population of Australian adults with stage 3-4 CKD. While a high ConI was predictive of the composite renal outcome in unadjusted models, neither WC nor ConI showed a significant association with kidney disease progression or mortality in adjusted models.

The outcome of this study, showing an inverse relationship between BMI and adverse outcomes, is similar to findings in previous studies investigating BMI and mortality in CKD patients [26-28]. An analysis of the Atherosclerosis Risk in Communities (ARIC) Study database demonstrated that a larger BMI was associated with better overall survival in a CKD population but an increased hazard of death in those without CKD [27]. Similarly, an evaluation of 12,534 individuals with stage 3-4. CKD participating in the Kidney Early Evaluation Program (KEEP) showed a survival advantage for patients who were obese, although the results were no longer significant when BMI exceeded $35 \mathrm{~kg} / \mathrm{m}^{2}$ [28]. In contrast, the study showed no association between BMI and rates of progression to end-stage kidney disease. In patients with stage 3-4 CKD pooled from the Atherosclerosis Risk in Communities and Cardiovascular Health Study [29], obese BMI was protective against a composite outcome of cardiovascular events, stroke and all-cause mortality.

The association of overweight BMI with better clinical outcomes may be accounted for by the inability of BMI to discriminate body composition. An elevated BMI has the potential to represent better overall nutrition and high muscle mass, and an increased ability to adapt to the protein energy wasting state commonly observed in kidney disease. Moreover, higher BMI has been shown as protective 
Table 1 Baseline characteristics of the stage 3-4 CKD study cohort based on BMI category

\begin{tabular}{|c|c|c|c|c|c|c|c|}
\hline \multirow[t]{3}{*}{ Variable } & \multirow{3}{*}{$\begin{array}{l}\text { Total } \\
\text { population } \\
(n=903)\end{array}$} & \multicolumn{6}{|c|}{ Breakdown by BMI $\left(\mathrm{kg} / \mathrm{m}^{2}\right)$} \\
\hline & & $<18.5$ & $18.5-24.9$ & $25-29.9$ & $30-39.9$ & $\geq 40$ & $p$-value* \\
\hline & & $(n=15 ; 1.8 \%)$ & $(n=174 ; 20.7 \%)$ & $(n=293 ; 34.8 \%)$ & $(n=288 ; 34.2 \%)$ & $(n=72 ; 8.6 \%)$ & \\
\hline Male gender & $515(57 \%)$ & $2(13.3 \%)$ & $96(55.2 \%)$ & $181(61.8 \%)$ & $169(58.7 \%)$ & $35(48.6 \%)$ & 0.002 \\
\hline Age (years) & $66.26 \pm 13.62$ & $56.5 \pm 18.8$ & $67 \pm 15.7$ & $68.3 \pm 13$ & $65.4 \pm 12.3$ & $61.0 \pm 11.1$ & $<0.001$ \\
\hline Race & & & & & & & 0.036 \\
\hline Caucasian & $708(78.4 \%)$ & $11(73.3 \%)$ & 137 (78.7 \%) & $241(82.3 \%)$ & $226(78.5 \%)$ & $51(70.8 \%)$ & \\
\hline Non-Caucasian & $115(12.7 \%)$ & $2(13.3 \%)$ & $29(16.6 \%)$ & $24(8.2 \%)$ & $36(12.5 \%)$ & $14(19.4 \%)$ & \\
\hline Not stated & $80(8.9 \%)$ & $2(13.3 \%)$ & $8(4.6 \%)$ & 28 (9.6 \%) & $26(9.0 \%)$ & 7 (9.7 \%) & \\
\hline Cause of CKD & & & & & & & $<0.001$ \\
\hline Diabetic nephropathy & $243(26.9 \%)$ & $0(0.0 \%)$ & $32(18.4 \%)$ & 59 (20.1\%) & $98(34.0 \%)$ & $33(45.8 \%)$ & \\
\hline Glomerulonephritis & 75 (8.3 \%) & $2(13.3 \%)$ & 17 (9.8 \%) & $23(7.8 \%)$ & 20 (6.9 \%) & $5(6.9 \%)$ & \\
\hline Cystic kidney disease & $26(2.9 \%)$ & $1(6.7 \%)$ & $7(4.0 \%)$ & $13(4.4 \%)$ & $4(1.4 \%)$ & $1(1.4 \%)$ & \\
\hline Other & $559(61.9 \%)$ & $12(80.0 \%)$ & $118(67.8 \%)$ & $198(67.6 \%)$ & $166(57.6 \%)$ & $33(45.8 \%)$ & \\
\hline \multicolumn{8}{|l|}{ Comorbidities } \\
\hline Diabetes mellitus $(n=902)$ & $387(42.9 \%)$ & $0(0.0 \%)$ & 49 (28.2 \%) & $114(28.9 \%)$ & $146(50.7 \%)$ & $47(66.2 \%)$ & $<0.001$ \\
\hline $\operatorname{CAD}(n=823)$ & $270(32.8 \%)$ & 1 (7.1\%) & $49(31.0 \%)$ & $93(33.6 \%)$ & $83(32.5 \%)$ & $21(33.9 \%)$ & 0.351 \\
\hline $\operatorname{CLD}(n=872)$ & $117(13.4 \%)$ & $1(6.7 \%)$ & $21(12.4 \%)$ & $38(13.5 \%)$ & $36(13.1 \%)$ & $16(22.5 \%)$ & 0.228 \\
\hline CBVD $(n=870)$ & $110(12.6 \%)$ & $0(0.0 \%)$ & $17(10.2 \%)$ & 39 (13.9 \%) & $40(14.2 \%)$ & $8(11.6 \%)$ & 0.393 \\
\hline $\operatorname{PVD}(n=854)$ & $140(16.4 \%)$ & $0(0.0 \%)$ & $20(12.2 \%)$ & $46(16.5 \%)$ & $54(19.8 \%)$ & $12(17.9 \%)$ & 0.115 \\
\hline \multicolumn{8}{|l|}{ Medication use } \\
\hline Lipid lowering $(n=839)$ & $402(47.9 \%)$ & $0(0.0 \%)$ & $72(43.4 \%)$ & $127(46.4 \%)$ & $143(54.2 \%)$ & $38(56.7 \%)$ & $<0.001$ \\
\hline ACEi/ARB $(n=839)$ & $509(60.7 \%)$ & $6(40.0 \%)$ & $93(56.0 \%)$ & $166(60.6 \%)$ & $168(63.6)$ & $50(74.6 \%)$ & 0.032 \\
\hline Antihypertensive ( $n=822)$ & $655(79.7 \%)$ & 8 (53.3 \%) & $125(75.8 \%)$ & 220 (80.9 \%) & 217 (82.2 \%) & $56(84.4 \%)$ & 0.033 \\
\hline $\mathrm{EPO}(n=839)$ & $29(3.5 \%)$ & $1(6.7 \%)$ & $8(4.8 \%)$ & 10 (3.6 \%) & $8(3.0 \%)$ & $2(3.0 \%)$ & 0.863 \\
\hline eGFR $(n=903)$ & $37.9 \pm 11.7$ & $38.3 \pm 12.2$ & $38.3 \pm 12.4$ & $37.4 \pm 11.3$ & $37.9 \pm 11.2$ & $39.9 \pm 12.0$ & 0.573 \\
\hline Stage 3 & $654(72.4 \%)$ & $11(1.8 \%)$ & 124 (20.2 \%) & $210(34.3 \%)$ & $212(34.6 \%)$ & 56 (9.1%) & \\
\hline Stage 4 & $249(27.6 \%)$ & $4(1.7 \%)$ & $50(21.8 \%)$ & 83 (36.2 \%) & 76 (33.2 \%) & $16(7.0 \%)$ & \\
\hline Proteinuria ( $n=780$ ) & & & & & & & 0.493 \\
\hline Microproteinuria & $459(58.8 \%)$ & $6(54.5 \%)$ & $88(59.5 \%)$ & $163(64.2 \%)$ & $138(54.3 \%)$ & $40(59.7 \%)$ & \\
\hline Macroproteinuria & $293(37.6 \%)$ & $5(45.5 \%)$ & $56(37.8 \%)$ & 83 (32.7 \%) & $104(40.9 \%)$ & $23(34.3 \%)$ & \\
\hline \multicolumn{8}{|l|}{ Obesity measures } \\
\hline WC $(n=594)$ & $101.6 \pm 16.1$ & $69.4 \pm 6.9$ & $86.1 \pm 8.2$ & $98.5 \pm 8.2$ & $111.2 \pm 10.3$ & $130.6 \pm 12.6$ & $<0.001$ \\
\hline Conl $(n=588)$ & $1.33 \pm 0.10$ & $1.21 \pm 0.11$ & $1.28 \pm 0.09$ & $1.33 \pm 0.09$ & $1.36 \pm 0.09$ & $1.39 \pm 0.09$ & $<0.001$ \\
\hline
\end{tabular}

Results expressed as mean \pm SD or number (percentage). The number of patients with data available follows the measured variable, if total population data not available

$C K D$ chronic kidney disease, $B M I$ body mass index, CAD coronary artery disease, $C L D$ chronic lung disease, CBVD cerebrovascular disease, $P V D$ peripheral vascular disease, $A C E i$ angiotensin-converting-enzyme inhibitor, $A R B$ angiotensin II receptor blocker, EPO erythropoietin, eGFR estimated glomerular filtration rate, WC waist circumference, Conl conicity index

*Differences between BMI categories were assessed by chi-squared test or ANOVA, depending on the data type

in proteinuric CKD but not in non-proteinuric CKD [30]. BMI is also unable to account for differences in body mass distribution and the risks associated with increased visceral adiposity. For example, an analysis by Panwar and colleagues [31] using the Reasons for Geographic and Racial Differences in Stroke (REGARDS) Study data showed a higher BMI was associated with lower ESRD risk in those without, but not those with the metabolic syndrome. In this analysis, controlling for WC, which was more likely to reflect abdominal fatness, did not appreciably alter the relationship between BMI and the composite renal outcome and only slightly attenuated the association with mortality. Furthermore, the Tehran Lipid and Glucose Study (TLGS) [32], found that increases in weight, BMI, WC and hip circumference were associated with reduced mortality in men but not women. In a gender-stratified analysis of this 


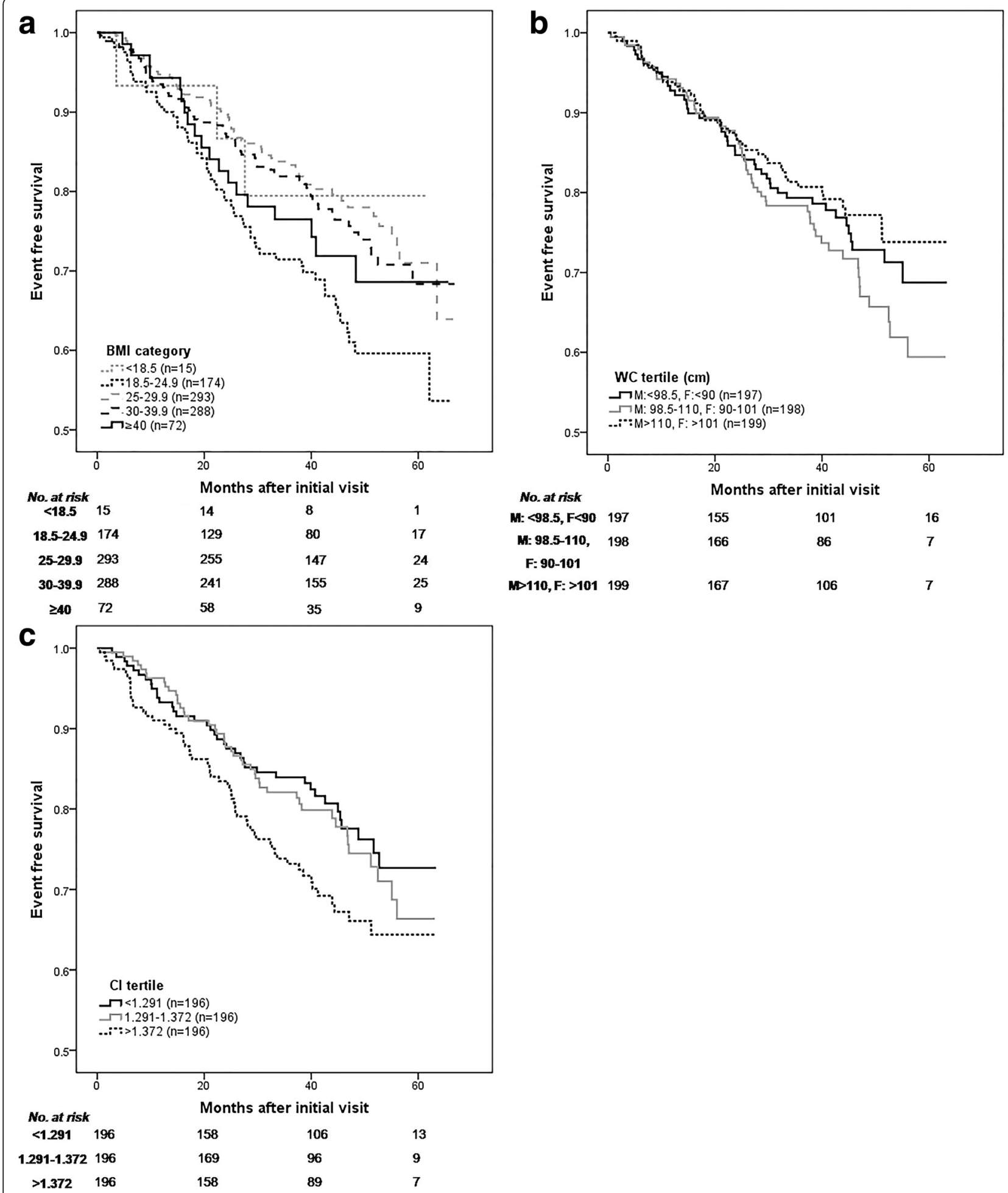

Fig. 2 Kaplan-Meier Curves for Progression to the Primary Outcome for Clinical Anthropometric Measures. The primary outcome included, doubling of serum creatinine, commencement of renal replacement therapy or all-cause mortality, with anthropometric measures of body mass index (BMI), waist circumference (WC) and conicity index (Conl). Shown below the graphs are the number of patients at risk. a. BMl by categories $\left(\mathrm{kg} / \mathrm{m}^{2}\right)$ [Log rank score 10.44, $p=0.034$ ]. b. WC by tertiles [Log rank score 3.09, $p=0.21$ ]. $\mathbf{c}$. Conl by tertiles [Log rank score 4.13, $p=0.13$ ] 
Table 2 Association between baseline obesity parameters and the primary outcome in stage 3-4 chronic kidney disease. Comparison performed using Cox proportional hazards modelling to compare body mass index, waist circumference and conicity index with the composite outcome of doubling of serum creatinine, commencement of renal replacement therapy or all-cause mortality

\begin{tabular}{|c|c|c|c|c|c|}
\hline & \multirow[b]{2}{*}{ No. (\%) } & \multicolumn{4}{|l|}{ HR $(95 \%$ Cl) } \\
\hline & & Crude & Model 1 & Model 2 & Model 3 \\
\hline \multicolumn{6}{|l|}{ Body mass index $\left(\mathrm{kg} / \mathrm{m}^{2}\right)$} \\
\hline$<18.5$ & $15(1.8)$ & $0.54(0.17-1.71)$ & $0.43(0.2-1.99)$ & $0.57(0.14-2.38)$ & $0.74(0.17-3.19)$ \\
\hline $18.5-24.9$ & $174(20.7)$ & 1.00 (referent) & 1.00 (referent) & 1.00 (referent) & 1.00 (referent) \\
\hline $25-29.9$ & $293(34.8)$ & $0.57(0.39-0.81)^{* *}$ & $0.55(0.38-0.79)^{* *}$ & $0.58(0.40-0.85)^{* *}$ & $0.50(0.33-0.75)^{* *}$ \\
\hline 30-39.9 & $288(34.2)$ & $0.63(0.44-0.90)^{*}$ & $0.65(0.46-0.93)^{*}$ & $0.71(0.49-1.03)$ & $0.62(0.41-0.93)^{*}$ \\
\hline$\geq 40$ & $72(8.6)$ & $0.74(0.44-1.24)$ & $0.82(0.49-1.39)$ & $0.96(0.56-1.65)$ & $0.94(0.52-1.69)$ \\
\hline \multicolumn{6}{|c|}{ Waist circumference tertiles $(\mathrm{cm})$} \\
\hline $\mathrm{M}:<98.5, \mathrm{~F}<90$ & $197(33.2)$ & 1.00 (referent) & 1.00 (referent) & 1.00 (referent) & 1.00 (referent) \\
\hline M: 98.5-110, F: 90-101 & $198(33.3)$ & $1.23(0.83-1.82)$ & $1.14(0.75-1.65)$ & $1.31(0.86-1.97)$ & $0.99(0.63-1.56)$ \\
\hline$M>110, F:>101$ & $199(33.5)$ & $0.87(0.57-1.33)$ & $0.89(0.58-1.35)$ & $1.00(0.64-1.57)$ & $0.87(0.54-1.41)$ \\
\hline \multicolumn{6}{|l|}{ Conicity index tertiles } \\
\hline$<1.291$ & $196(33.3)$ & 1.00 (referent) & 1.00 (referent) & 1.00 (referent) & 1.00 (referent) \\
\hline $1.291-1.372$ & $196(33.3)$ & $1.14(0.74-1.80)$ & $1.03(0.67-1.59)$ & $1.03(0.65-1.63)$ & $1.13(0.68-1.86)$ \\
\hline$>1.372$ & $196(33.3)$ & $1.59(1.05-2.39)^{*}$ & $1.46(0.96-2.20)$ & $1.39(0.88-2.22)$ & $1.16(0.69-1.95)$ \\
\hline
\end{tabular}

Results expressed as number (percentage) and hazard ratio (95\% confidence interval)

Model 1: Adjusted for age

Model 2: Adjusted for age, gender, race (Caucasian vs. Non-Caucasian)

Model 3: Model $2+$ estimated glomerular filtration rate, proteinuria, cause of chronic kidney disease, diabetes status

${ }^{*} P \leq 0.05,{ }^{*} P \leq 0.01$

data the U-shaped association between BMI and outcomes was found to be more pronounced in men than women but otherwise trends were maintained.

In contrast to the findings of the current study, Evans et al. [33] found that BMI was unrelated to time to RRT commencement in a Swedish population of 920 CKD patients, although the inverse relationship with mortality was maintained. Using data from the Modification of Diet in Renal Disease (MDRD) study [34], Madero et al. were also unable to find an independent association between BMI and mortality. However, in contrast to the population in the present study, the MDRD study excluded a number of important conditions that may have been impacted by the presence of obesity (including type 1 diabetes, insulin-dependent type 2 diabetes, autoimmune glomerulonephritis and renal artery stenosis) and potentially played a role in the different outcomes of the two studies.

Due to the limitations of BMI as a measure of obesity, it was hypothesised that more specific anthropometric measures of central obesity, including WC and ConI, may be better prognostic indicators than BMI. However, neither WC nor ConI were independent risk factors for kidney disease progression or death. This finding contrasts with that of a post-hoc analysis of 5,805 stage 1-4 CKD patients participating in the REGARDS Study, which showed that the highest WC group had an approximately two-fold increased hazard rate for all-cause mortality [19]. The apparent disparity in findings may be partly explained by the differences in the study populations. The REGARDS cohort was made up of more than $50 \%$ individuals with stage 1-2 CKD, whilst the present study cohort was solely comprised of patients with stage 3 or 4 CKD. Participants categorised with very early CKD (eGFR $\geq 60 \mathrm{~mL} / \mathrm{min}$ / $1.73 \mathrm{~m}^{2}$ ) therefore may have been more closely matched to the risk profile of the general population than individuals with more advanced disease, explaining the deleterious effects of higher WC seen in their study. Furthermore, there is conflicting evidence surrounding the reliability of WC as a marker for visceral adiposity in CKD. Several studies have found different levels of correlation between visceral fat and measured WC.

In kidney disease especially, these inconsistent results may relate to the fluid disturbances which would alter the relationship between abdominal girth and visceral fat. Furthermore, there are methodological issues with the reproducibility of WC in the real world setting, more so than for BMI [35]. Panoulas and colleagues highlighted this problem in a study of the intra- and inter-operator variability of WC measurements, where there were significant differences between measurements by health practitioners [36]. While measurements in the current study were all recorded using a standardised protocol, there were a number of operators involved. The lack of significant correlation between WC and outcomes may indicate that the utility of WC is limited in real world settings. 


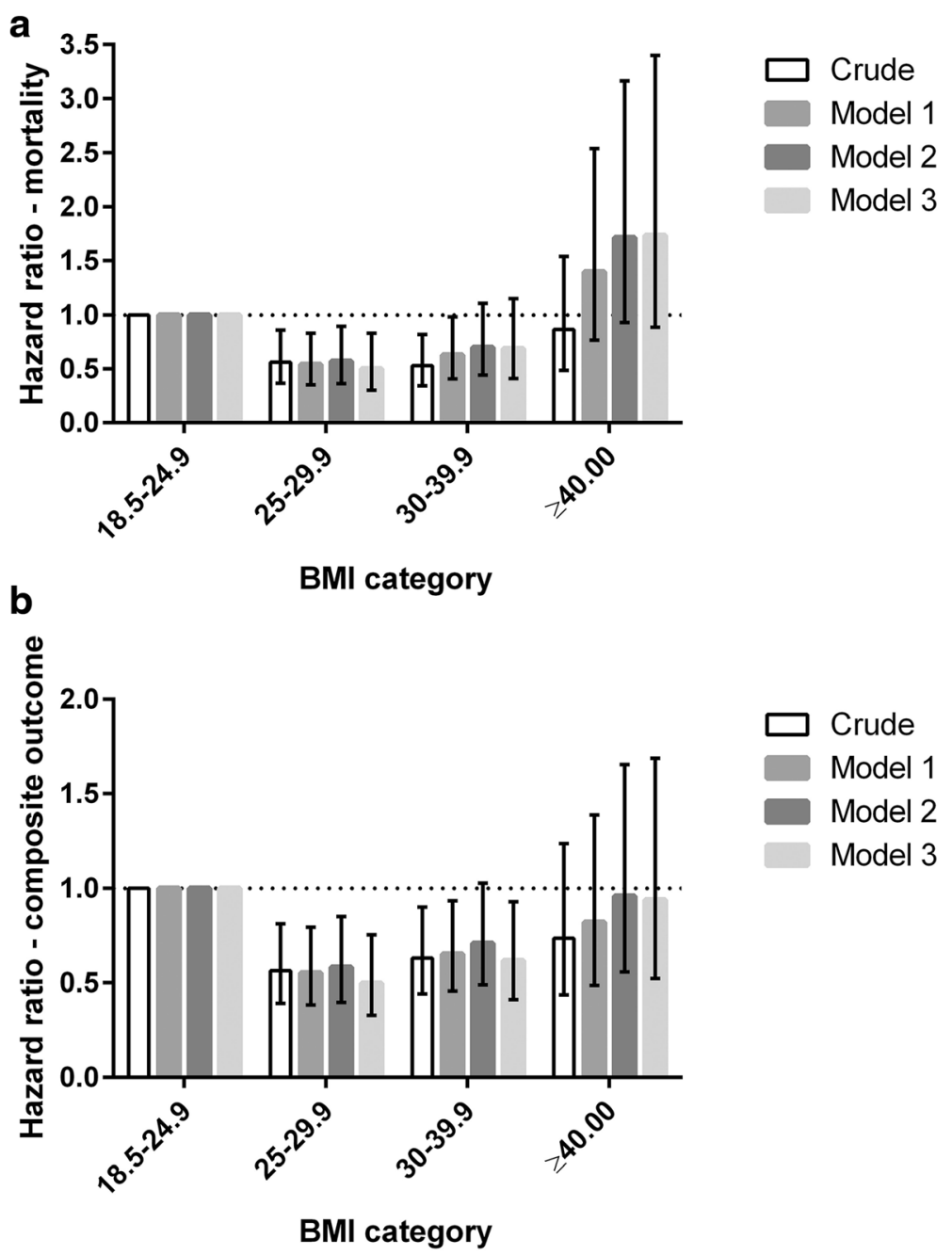

Fig. 3 Hazard Ratios for the Composite Outcome (a) and Mortality (b) for BMI Categories. A U-shaped association is evident between BMI categories and hazard ratios for both the composite outcome and mortality, in stage 3-4 CKD patients. Hazard ratios with $95 \%$ Cls shown for Cox regression models: crude, model 1 (age-adjusted), model 2 (model 1 plus gender and race-adjusted), model 3 (model 2 plus estimated glomerular filtration rate, proteinuria, cause of chronic kidney disease, diabetes status)

ConI was also found to not be independently predictive of kidney disease progression or death in patients with stages 3-4 CKD. Although there have been no prior studies of ConI as a prognostic factor in nondialysis CKD patients, Evans et al. reported that ConI was more strongly correlated with risk factors for cardiovascular disease and CKD progression, including eGFR, proteinuria, uric acid and systolic blood pressure, than WC or BMI [20]. A small study of 104 predialysis CKD patients found that increasing ConI was associated with greater eGFR reduction over a 12 month period [21]. Furthermore, a study of 173 haemodialysis patients demonstrated an association between elevated ConI and mortality, which was no longer apparent following adjustment for markers of inflammation and malnutrition [18].
This study has a number of important limitations. Firstly, the observational design meant that a causal relationship could not be inferred from the observed associations. Secondly, the small sample size and limited study duration (median follow-up 3.23 years) meant that the possibility of a type 2 statistical error could not be excluded, particularly in the extreme BMI categories $\left(<18.5\right.$ and $\left.\geq 40 \mathrm{~kg} / \mathrm{m}^{2}\right)$. Nevertheless, 903 individuals participated in the study and 224 (25\%) experienced a primary event. Thirdly, there was limited adjustment for comorbidities in the models, such that the possibility of residual confounding could not be excluded. Fourthly, while there was a standard protocol used to measure waist circumference by trained health practitioners, the risk of inter-observer variation was not formally evaluated. Fifthly, the assessment of abdominal girth may have been confounded in patients with polycystic 
Table 3 Association between baseline obesity parameters and all-cause mortality in stage 3-4 chronic kidney disease. Comparison performed using Cox proportional hazards modelling to compare body mass index, waist circumference and conicity index with all-cause mortality

\begin{tabular}{|c|c|c|c|c|c|}
\hline & \multirow[b]{2}{*}{ No. (\%) } & \multicolumn{4}{|l|}{ HR $(95 \% \mathrm{Cl})$} \\
\hline & & Crude & Model 1 & Model 2 & Model 3 \\
\hline \multicolumn{6}{|l|}{ Body mass index $\left(\mathrm{kg} / \mathrm{m}^{2}\right)$} \\
\hline$<18.5$ & $15(1.8)$ & $0.23(0.03-1.67)$ & $0.39(0.05-2.89)$ & $0.49(0.07-3.58)$ & $1.03(0.14-7.80)$ \\
\hline $18.5-24.9$ & $174(20.7)$ & 1.00 (referent) & 1.00 (referent) & 1.00 (referent) & 1.00 (referent) \\
\hline $25-29.9$ & $293(34.8)$ & $0.56(0.37-0.86)^{* *}$ & $.54(0.35-0.83)^{* *}$ & $0.57(0.36-0.90)^{*}$ & $0.50(0.30-0.83)^{* *}$ \\
\hline $30-39.9$ & $288(34.2)$ & $0.53(0.34-0.82)^{* *}$ & $.63(0.41-0.98)^{*}$ & $0.70(0.44-1.11)$ & $0.69(0.41-1.15)$ \\
\hline$\geq 40$ & $72(8.6)$ & $0.86(0.49-1.54)$ & $1.40(0.77-2.54)$ & $1.71(0.93-3.16)$ & $1.74(0.89-3.40)$ \\
\hline \multicolumn{6}{|c|}{ Waist circumference tertiles $(\mathrm{cm})$} \\
\hline $\mathrm{M}:<98.5, \mathrm{~F}<90$ & $197(33.2)$ & 1.00 (referent) & 1.00 (referent) & 1.00 (referent) & 1.00 (referent) \\
\hline M: 98.5-110, F: 90-101 & $198(33.3)$ & $1.21(0.75-1.95)$ & $1.04(0.65-1.68)$ & $1.18(0.72-1.95)$ & $0.90(0.52-1.58)$ \\
\hline$M>110, F:>101$ & $199(33.5)$ & $0.83(0.50-1.40)$ & $1.07(0.59-1.71)$ & $1.09(0.62-1.92)$ & $1.00(0.54-1.86)$ \\
\hline \multicolumn{6}{|l|}{ Conicity index tertiles } \\
\hline$<1.291$ & $196(33.3)$ & 1.00 (referent) & 1.00 (referent) & 1.00 (referent) & 1.00 (referent) \\
\hline $1.291-1.372$ & $196(33.3)$ & $1.61(0.93-2.77)$ & $1.29(0.75-2.22)$ & $1.33(0.74-2.36)$ & $1.67(0.89-3.13)$ \\
\hline$>1.372$ & 196 (33.3) & $1.85(1.09-3.15)^{*}$ & $1.57(0.92-2.67)$ & $1.53(0.84-2.79)$ & $1.31(0.66-2.58)$ \\
\hline
\end{tabular}

Results expressed as number (percentage) and hazard ratio (95\% confidence interval) Model 1: Adjusted for age

Model 2: Adjusted for age, gender, race (Caucasian vs. Non-Caucasian)

Model 3: Model $2+$ estimated glomerular filtration rate, proteinuria, cause of chronic kidney disease, diabetes status ${ }^{*} P \leq 0.05,{ }^{* *} P \leq 0.01$

kidney disease, although such patients comprised less than $3 \%$ of the total population. Sixthly, WC and ConI data were missing in approximately one-third of patients, which may have introduced bias. Finally, there were very few Aboriginal or Torres Strait Islander peoples within the study cohort, such that the results of the current investigation may not be generalisable to this high risk population [37].

\section{Conclusion}

This study demonstrated that, in an Australian stage 3-4 CKD population, $\mathrm{BMI}$ in the overweight and obesity classes $\mathrm{I} / \mathrm{II}$ range is associated with reduced risks of progression of renal disease and mortality, and that adjusting for WC does not significantly alter this association. Alternative anthropometric measures of central obesity (WC and ConI) were not significantly associated with disease progression or mortality in this population, however BMI may be valuable for risk-stratification of newly referred CKD patients.

\section{Additional file}

Additional file 1: Table S1. Association between baseline obesity parameters and the primary outcome in stage 3-4 chronic kidney disease stratified by gender. Comparison performed using Cox proportional hazards modelling to compare body mass index, waist circumference and conicity index with the composite outcome of doubling of serum creatinine, commencement of renal replacement therapy or all-cause mortality. Table S2. Association between baseline obesity parameters and all-cause mortality in stage 3-4 chronic kidney disease stratified by gender. Comparison performed using Cox proportional hazards modelling to compare body mass index, waist circumference and conicity index with all-cause mortality by gender. (DOCX $24 \mathrm{~kb}$ )

\section{Abbreviations}

ACR, Albumin:creatinine ratio; ANZDATA, Australia and New Zealand Dialysis and Transplant; BMI, Body mass index; CKD, Chronic kidney disease; CKD-EPI, Chronic Kidney Disease Epidemiology Collaboration; Conl, Conicity index; CVD, Cardiovascular disease; eGFR, Estimated glomerular filtration rate; HR, Hazard ratio; IQR, Interquartile range; MDRD, Modification of Diet in Renal Disease; PAH, Princess Alexandra Hospital; PCR, Protein:creatinine ratio; RRT, Renal replacement therapy; WC, Waist circumference

\section{Acknowledgements}

KL. Campbell is supported by a Queensland Government, Health and Medical Research (HMR) Health Research Fellowship, and a Lions Senior Medical Research Fellowship. DW. Johnson is also supported by a

Queensland Government HMR Health Research Fellowship.

\section{Funding}

No specific funding was obtained for this study.

Availability of data and materials

All data supporting the study is presented in the manuscript or available upon request from the corresponding author.

\section{Authors' contributions}

ED was involved in data collection, gaining ethical approval and completing the statistical analysis. KC assisted in the study design, ethical approval, data collection and statistical analysis. GG was involved in the study design and interpretation of findings. $\mathrm{CH}$ assisted with the statistical analysis and interpretation. $\mathrm{NI}$ contributed to study design, ethical approval and analysis of results. DJ was involved in study design, assisted with statistical analysis and interpretation. All authors contributed to drafting of the manuscript and approved of the final copy. 


\section{Competing interests}

The authors of this manuscript confirm that they have no competing interest to declare.

\section{Consent to publish}

Not applicable.

\section{Ethics and consent to participate}

Ethics approval was granted through Metro South Human Research Ethics Committee (HREC/10/QPAH/71) and University of Queensland School of Medicine Low Risk Ethics Committee (2014-SOMILRE-0094). Patient consent was attained for use of clinical data in the Princess Alexandra Hospital (PAH) Nephrology Database.

\section{Author details}

'Centre for Kidney Disease Research, School of Medicine, University of Queensland, Translational Research Institute, Brisbane, QLD, Australia. ${ }^{2}$ Department of Nephrology, Princess Alexandra Hospital, Brisbane, QLD, Australia. ${ }^{3}$ Australasian Kidney Trials Network, University of Queensland, Brisbane, Australia.

\section{Received: 4 August 2015 Accepted: 14 June 2016}

\section{Published online: 08 July 2016}

\section{References}

1. Coresh J, Selvin E, Stevens LA, Manzi J, Kusek JW, Eggers P, et al. Prevalence of chronic kidney disease in the United States. JAMA. 2007;298(17):2038-47.

2. White SL, Polkinghorne KR, Atkins RC, Chadban SJ. Comparison of the prevalence and mortality risk of CKD in Australia using the CKD epidemiology collaboration (CKD-EPI) and modification of diet in renal disease (MDRD) study GFR estimating equations: the AusDiab (Australian diabetes, obesity and lifestyle) study. Am J Kidney Dis. 2010;55(4):660-70.

3. Johnson DW, Atai E, Chan M, Phoon RK, Scott C, Toussaint ND, et al. KHACARI guideline: early chronic kidney disease: detection, prevention and management. Nephrology (Carlton). 2013;18(5):340-50.

4. Stevens PE, Levin A. Evaluation and management of chronic kidney disease: synopsis of the kidney disease: improving global outcomes 2012 clinical practice guideline. Ann Intern Med. 2013;158(11):825-30.

5. National Kidney Foundation. KDOQI clinical practice guidelines and clinical practice recommendations for diabetes and chronic kidney disease. Am J Kidney Dis. 2007:49(2, Suppl 2):S12-154.

6. Fox CS, Larson MG, Leip EP, Culleton B, Wilson PW, Levy D. Predictors of new-onset kidney disease in a community-based population. JAMA. 2004; 291(7):844-50.

7. Wändell PE, Carlsson AC, Theobald $\mathrm{H}$. The association between BMI value and long-term mortality. Int J Obes. 2009;33(5):577-82.

8. Lu JL, Kalantar-Zadeh K, Ma JZ, Quarles LD, Kovesdy CP. Association of body mass index with outcomes in patients with CKD. J Am Soc Nephrol. 2014; 25(9):2088-96.

9. Flegal KM, Graubard BI, Williamson DF, Cooper RS. Reverse causation and illness-related weight loss in observational studies of body weight and mortality. Am J Epidemiol. 2011;173(1):1-9.

10. Agarwal R, Bills JE, Light RP. Diagnosing obesity by body mass index in chronic kidney disease: an explanation for the "obesity paradox?". Hypertension. 2010;56(5):893-900.

11. Romero-Corral A, Somers VK, Sierra-Johnson J, Thomas RJ, Collazo-Clavell ML, Korinek J, et al. Accuracy of body mass index in diagnosing obesity in the adult general population. Int J Obes. 2008;32(6):959-66.

12. Liu J, Fox CS, Hickson DA, May WD, Hairston KG, Carr JJ, et al. Impact of abdominal visceral and subcutaneous adipose tissue on cardiometabolic risk factors: the Jackson heart study. J Clin Endocrinol Metab. 2010;95(12):5419-26.

13. Cnop M, Havel PJ, Utzschneider KM, Carr DB, Sinha MK, Boyko EJ, et al. Relationship of adiponectin to body fat distribution, insulin sensitivity and plasma lipoproteins: evidence for independent roles of age and sex. Diabetologia. 2003;46(4):459-69.

14. Valdez R, Seidell JC, Ahn YI, Weiss KM. A new index of abdominal adiposity as an indicator of risk for cardiovascular disease. A cross-population study. Int J Obes Relat Metab Disord. 1993;17(2):77-82.

15. Janssen I, Katzmarzyk PT, Ross R. Waist circumference and not body mass index explains obesity-related health risk. Am J Clin Nutr. 2004;79(3):379.
16. Navaneethan SD, Kirwan JP, Arrigain S, Schreiber MJ, Sarnak MJ, Schold JD Obesity, anthropometric measures and chronic kidney disease complications. Am J Nephrol. 2012;36(3):219-27.

17. Postorino M, Marino C, Tripepi G, Zoccali C, Credit Working Group. Abdominal obesity and all-cause and cardiovascular mortality in end-stage renal disease. JACC. 2009;53(15):1265-72.

18. Cordeiro AC, Qureshi AR, Stenvinkel P, Heimbürger $O$, Axelsson J, Bárány P, et al. Abdominal fat deposition is associated with increased inflammation, protein-energy wasting and worse outcome in patients undergoing haemodialysis. Nephrol Dial Transplant. 2010;25(2):562-8.

19. Kramer H, Shoham D, McClure LA, Durazo-Arvizu R, Howard G, Judd S, et al. Association of waist circumference and body mass index with all-cause mortality in CKD: the REGARDS (reasons for geographic and racial differences in stroke) study. Am J Kidney Dis. 2011;58(2):177-85.

20. Evans PD, Mclntyre NJ, Fluck RJ, McIntyre CW, Taal MW. Anthropomorphic measurements that include central fat distribution are more closely related with key risk factors than BMI in CKD stage 3. PLoS One. 2012;7(4):e34699.

21. Sezer S, Karakan S, Acar NO. Association of conicity index and renal progression in pre-dialysis chronic kidney disease. Ren Fail. 2012;34(2):165-70.

22. Ruperto M, Barril G, Sánchez-Muniz FJ. Conicity index as a contributor marker of inflammation in haemodialysis patients. Nutr Hosp. 2013;28(5):1688-95.

23. Levey AS, Stevens LA, Schmid CH, Zhang YL, Castro 3rd AF, Feldman $\mathrm{HI}$, et al. A new equation to estimate glomerular filtration rate. Ann Intern Med. 2009;150(9):604-12.

24. Johnson DW, Harvie B, Hobbs T, et al. Chronic kidney disease management in general practice. Adelaide: Kidney Health Australia; 2012.

25. Haynes R, Staplin N, Emberson J, Herrington WG, Tomson C, Agodoa L, et al. Evaluating the contribution of the cause of kidney disease to prognosis in CKD: results from the study of heart and renal protection (SHARP). Am J Kidney Dis. 2014;64(1):40-8.

26. Kovesdy CP, Anderson JE, Kalantar-Zadeh K. Paradoxical association between body mass index and mortality in men with CKD not yet on dialysis. Am J Kidney Dis. 2007:49(5):581-91.

27. Kwan BCH, Murtaugh MA, Beddhu S. Associations of body size with metabolic syndrome and mortality in moderate chronic kidney disease. CJASN. 2007;2(5):992-8.

28. Babayev R, Whaley-Connell A, Kshirsagar A, Klemmer P, Navaneethan S, Chen SC, et al. Association of race and body mass index with ESRD and mortality in CKD stages 3-4: results from the kidney early evaluation program (KEEP). Am J Kidney Dis. 2013;61(3):404-12.

29. Elsayed EF, Tighiouart H, Weiner DE, Griffith J, Salem D, Levey AS, et al. Waist-to-Hip ratio and body mass index as risk factors for cardiovascular events in CKD. Am J Kidney Dis. 2008;52(1):49-57.

30. Obermayr RP, Temml C, Gutjahr G, Kainz A, Klauser-Braun R, Függer R, et al. Body mass index modifies the risk of cardiovascular death in proteinuric chronic kidney disease. Nephrol Dial Transplant. 2009;24(8):2421-8.

31. Panwar B, Bhupesh P, Lynae JH, Rikki MT, Paul M. Obesity, metabolic health, and the risk of end-stage renal disease. Kidney Int. 2015;87(6):1216-22.

32. Mohebi R, Simforoosh A, Tohidi M, Azizi F. Obesity paradox and risk of mortality events in chronic kidney disease patients: a decade of follow-up in Tehran lipid and glucose study. J Ren Nutr. 2015;25(4):345-50.

33. Evans M, Fryzek JP, Elinder CG, Cohen SS, McLaughlin JK, Nyrén O, et al. The natural history of chronic renal failure: results from an unselected, populationbased, inception cohort in Sweden. Am J Kidney Dis. 2005;46(5):863-70.

34. Madero M, Sarnak MJ, Wang X, Sceppa CC, Greene T, Beck GJ, et al. Body mass index and mortality in CKD. Am J Kidney Dis. 2007;50(3):404-11.

35. Nadas J, Putz Z, Kolev G, Nagy S, Jermendy G. Intraobserver and interobserver variability of measuring waist circumference. Med Sci Monit. 2008;14(1):Cr15-8.

36. Panoulas VF, Ahmad N, Fazal AA, Kassamali RH, Nightingale P, Kitas GD, et al. The inter-operator variability in measuring waist circumference and its potential impact on the diagnosis of the metabolic syndrome. Postgrad Med J. 2008;84(993):344-7.

37. Hoy WE, Hughson MD, Singh GR, Douglas-Denton R, Bertram JF. Reduced nephron number and glomerulomegaly in Australian aborigines: a group at high risk for renal disease and hypertension. Kidney Int. 2006;70(1):104-10. 